\title{
Ozone layer: the road not taken
}

SIR - As distinguished and experienced participants in the evolution of scientific understanding of ozone-layer depletion, Prather et al. (Nature 381, 551-554; 1996) provide a valuable quantitative perspective on the advantages of acting early to correct global atmospheric catastrophe. Although scientists have given an early warning on climate change, there has been little success so far in slowing the growth in greenhouse gas emissions. It is therefore worth looking a little more deeply at some of the reasons why protection of the ozone layer was so successful.

The 1996 phase-out of chlorofluorocarbons (CFCs) and other ozone-depleting substances (ODSs) under the Montreal Protocol is economically and environmentally successful for four reasons. First, scientists ultimately persuaded industry of the need for prompt action. Second, governments selected flexible, market-based regulation. Third, industry cooperated to speed development and selection of new technology. Finally, industry helped policy-makers to choose schedules that were technically feasible and allowed time for wise choice.

In retrospect, it can be seen that more science, earlier, could have given even greater warning that CFCs destroy the ozone layer. This would have reduced dependence on ODSs and saved the cost of eliminating them. The importance of science in protecting the ozone layer is strong justification for continued financing of basic atmospheric research. If science had been less well funded, scientists less proactive or diplomats less successful, signing of the protocol might have been delayed. Moreover, the cost of the ODS phase-out would have been about half as much if CFC manufacturers had been less successful in their political interventions between 1974 and 1987. Much of the chlorine in the atmosphere is from applications such as cosmetic products for which there were technically and economically feasible alternatives a decade before the protocol was signed and two decades before the ODS phase-out.

Prather et al. hypothesize on what might have happened if the phase-out had started later but, to protect the ozone layer, industry had had to achieve the same phase-out schedule. With a global emergency, elimination of many ODS applications with identified alternatives could have been significantly accelerated with small increases in ultimate costs. Such uses include aerosol

\section{corres@nature.com}

Letters submitted for Correspondence should be typed, double-spaced, on one side of the paper only, or e-mailed to corres@nature.com products, metal cleaning, rigid-foam building insulation, flexible foam and many halon applications. In some cases, alternatives and substitutes were quickly identified and could have been more quickly implemented under emergency conditions. Such uses include HFC-134a for automobile air-conditioning and refrigeration; hydrochlorofluorocarbon (HCFC) substitutes for refrigeration and air-conditioning, sterilization and rigid foams; and aqueous cleaning of electronic assemblies. In other cases, five years or more of well-funded, aggressive efforts were required to identify new technology for critical applications that had no alternatives and substitutes in 1987. If work on alternatives had not begun in 1987, ODS use would have had to continue after 1996. Such uses include aerospace applications such as electrical, optical and precision cleaning of both civilian aircraft and military weapons.

The most serious consequence of a later start on ozone-layer protection would probably have been to owners of automobiles, refrigeration equipment and airconditioners which now depend on recycled CFCs. Recycled CFCs are inexpensive, and retrofit kits are just entering the market for some equipment.

A late start would also have required industry and consumers to select from among the very first technologies available. Some of the most environmentally acceptable, cost-effective substitutes were second-generation replacements for CFCs. Much CFC-113 solvent use in electronics manufacturing would have been replaced with HCFCs, chlorinated solvents and alcohols instead of technically and environmentally superior no-clean soldering. Moreover, industry is less likely to finance continuing research and development once a technology is selected.

The protocol phase-out schedule has maintained public confidence in the value of the changes that are being made; a later start might have been too expensive to consumers and some companies to maintain necessary political support. Even now, there is concern that developed countries will fail to meet their obligations for financing the phase-out in developing countries.

In sum, the Montreal Protocol was none too early, hopefully just in time, but possibly a little too late.

\section{Stephen 0. Andersen}

Stratospheric Protection Division, Environmental Protection Agency, 401 M Street SW,

Washington, DC 20460, USA

Alan Miller

Center for Global Change,

University of Maryland,

College Park, Maryland 20740, USA

\section{Counterattack}

SIR - In his review of Confronting the Experts (Nature 382, 35-36; 1996), Walter Gratzer selected my chapter for the harshest criticism. The reason he gave was his assertion that I believe "for instance that all attempts at cellular fractionation generate nothing but artefacts, that practically everything revealed by the electron microscope is an artefact of fixation and staining, that there is no such thing as a lipid bilayer membrane, that the known cytoplasmic granules are mirages, the nervous system tissue consists of granular "ground substance' with 'naked nuclei'...”.

Each of these five statements as quoted is such a gross distortion of my views that $\mathrm{I}$ can only conclude that Gratzer has not read any of my original publications on these topics. I might have expected that a senior colleague would have read the evidence upon which my conclusions are based before condemning them.

In passing, he remarks that my finding that ATP is hydrolysable by visible light could not be true because it does not absorb. Perhaps he does not know that colourless solutions of adrenaline, lactate, fumarate, hydrogen peroxide, scintillation fluids - and, yes, ATP - are stored in dark bottles because they are unstable in visible light.

\section{Harold Hillman}

Unity Laboratory of Applied Neurobiology, 76 Epsom Road,

Guildford, Surrey GU1 2BX, UK

\section{Manners please}

SIR - Why are academics rude to one another? Discussion of opposing views so often degenerates into name-calling. An investigator new to a field will make many personal discoveries, some of which were learnt in kindergarten by the expert. The expert, however, should not launch a personal attack on the novice for verbalizing such ideas. Indeed, it is always possible that a fresh mind, with a new perspective on a problem, will make suggestions previously overlooked. Criticism aimed at the person rather than the issue in hand serves no good purpose. It is a poor reflection on the perpetrator, since it indicates frustration and an inability to make further constructive comment. Harsh words generate alienation and discouragement. We all make mistakes from time to time, so a sympathetic attitude would be of universal benefit.

\section{Valerie Good}

Centre for Mechanisms of Human Toxicity, University of Leicester,

Hodgkin Building,

Lancaster Road,

Leicester, LE2 2AB, UK 